\title{
Author Index Volume 38 (2014)
}

The issue number is given in front of the pagination

Acosta-Cabronero, J., see Diaz-de-Grenu, L.Z. (3) 647-659

Adolfsson, O., see Paganetti, P. (4) 767-786

Albani, D., see Benussi, L. (4) 799-808

Alberoni, M., see Saresella, M. (2) 403-413

Albert, M., see Razlighi, Q.R. (3) 661-668

Alexandrova, I., see Bobkova, N.V. (2) 425-435

Altamura, C., see Scrascia, F. (4) 939-950

Altavilla, R., see Scrascia, F. (4) 939-950

Anderson, M.E., see Xu, W. (3) 621-632

Anfossi, M., R. Colao, M. Gallo, L. Bernardi, M.E. Conidi, F. Frangipane, F. Vasso, G. Puccio, A. Clodomiro, M. Mirabelli, S.A.M. Curcio, G. Torchia, N. Smirne, R. Di Lorenzo, R. Maletta and A.C. Bruni, Identification of Three Novel LRRK2 Mutations associated with Parkinson's Disease in a Calabrian Population (2) 351-357

Anghinah, R., see de Souza Rolim, T. (4) 823-829

Antoniello, K., see Paganetti, P. (4) 767-786

Arancio, O., see Lee, L. (1) 49-62

Arisi, I., see Tzanoulinou, S. (4) 951-964

Auckland, L., see Parham, C. (2) 415-423

Baglio, F., see Mancuso, R. (4) 741-745

Bähr, M., see Schmitz, M. (3) 551-565

Bahri, S., see Chien, D.T. (1) 171-184

Bai, Z., see Wang, J. (2) 245-250

Baker, M., see Taher, N. (4) 831-844

Baldauf-Wagner, S., see Shinto, L. (1) 111-120

Bartolini, M., see Buratti, L. (2) 445-453

Bauer, A., see Kirste, T. (1) 121-132

Bazenet, C., see Kiddle, S.J. (3) 515-531

Belleville, S., see Peters, F. (2) 307-318

Ben-David, B.M., A. Tewari, V. Shakuf and P.H.H.M. Van Lieshout, Stroop Effects in Alzheimer's Disease: Selective Attention Speed of Processing, or Color-naming? A Meta-Analysis (4) 923-938

Bendini, M., see Gallucci, M. (3) 581-587

Benussi, L., G. Rossi, M. Glionna, E. Tonoli, E. Piccoli, S. Fostinelli, A. Paterlini, R. Flocco, D. Albani, R. Pantieri, C. Cereda , G. Forloni, F. Tagliavini, G. Binetti and R. Ghidoni, C9ORF72 Hexanucleotide Repeat Number in Frontotempo- ral Lobar Degeneration: A Genotype-Phenotype Correlation Study (4) 799-808

Benussi, L., see Ghidoni, R. (3) 533-539

Bernardi, L., see Anfossi, M. (2) 351-357

Bernick, C., see Cummings, J. (1) 103-109

Bertolucci, P.H., see Silva, P.N. (1) 165-170

Biessels, G.J., see Heringa, S.M. (1) 211-221

Binetti, G., see Benussi, L. (4) 799-808

Binetti, G., see Ghidoni, R. (3) 533-539

Bix, G., see Parham, C. (2) 415-423

Blacker, D., see Razlighi, Q.R. (3) 661-668

Bobkova, N.V., D.G. Garbuz, I. Nesterova, N. Medvinskaya, A. Samokhin, I. Alexandrova, V. Yashin, V. Karpov, M.S. Kukharsky, N.N. Ninkina, A.A. Smirnov, E. Nudler and M. Evgen'ev, Therapeutic Effect of Exogenous Hsp70 in Mouse Models of Alzheimer's Disease (2) 425-435

Boldrini, P., see Gallucci, M. (3) 581-587

Bolitho, S., see Naismith, S.L. (4) 867-879

Bonakis, A., N.-T. Economou, T. Paparrigopoulos, E. Bonanni, M. Maestri, L. Carnicelli, E.D. Coscio, P. Ktonas, E. Vagiakis, P. Theodoropoulos and S.G. Papageorgiou, Sleep in Frontotemporal Dementia is Equally or Possibly More Disrupted, and at an Earlier Stage, When Compared to Sleep in Alzheimer's Disease (1) 85-91

Bonanni, E., see Bonakis, A. (1) 85-91

Borghys, H., T. Jacobs, B.V. Broeck, L. Dillen, D. Dhuyvetter, H. Gijsen and M. Mercken, Comparison of Two Different Methods for Measurement of Amyloid- $\beta$ Peptides in Cerebrospinal Fluid after BACE1 Inhibition in a Dog Model (1) $39-48$

Bossers, K., see Wirz, K.T.S. (4) 719-740

Bourdette, D., see Shinto, L. (1) 111-120

Bozzali, M., see Serra, L. (2) 391-402

Braga, I.L., see Silva, P.N. (1) 165-170

Branch, N., see Maruszak, A. (1) 11-38

Brandi, R., see Tzanoulinou, S. (4) 951-964

Brandt, J., see Razlighi, Q.R. (3) 661-668

Broeck, B.V., see Borghys, H. (1) 39-48

Broersen, L.M., see van Wijk, N. (3) 459-479

Bruni, A.C., see Anfossi, M. (2) 351-357 
Bumgarner, L., see Shinto, L. (1) 111-120

Buratti, L., G. Viticchi, L. Falsetti, C. Cagnetti, S. Luzzi, M. Bartolini, L. Provinciali and M. Silvestrini, Vascular Impairment in Alzheimer's Disease: The Role of Obstructive Sleep Apnea (2) 445-453

Bush, A.I., see Squitti, R. (4) 809-822

Busija, D.W., see Carvalho, C. (1) 75-83

Cabinio, M., see Mancuso, R. (4) 741-745

Cagnetti, C., see Buratti, L. (2) 445-453

Cai, H., see Yan, X.-X. (4) 705-718

Calabrese, E., see Mancuso, R. (4) 741-745

Calabrese, E., see Saresella, M. (2) 403-413

Calissano, P., see Severini, C. (2) 281-293

Caltagirone, C., see Serra, L. (2) 391-402

Cano-Cuenca, N., J.E. Solís-García del Pozo and J. Jordán, Evidence for the Efficacy of Latrepirdine (Dimebon) Treatment for Improvement of Cognitive Function: A Meta-Analysis (1) 155-164

Cantello, R., see Carecchio, M. (4) 747-752

Capsoni, S., see Tzanoulinou, S. (4) 951-964

Carecchio, M., D. Galimberti, C. Fenoglio, M. Serpente, E. Scarpini, C. Comi, E. Terazzi and R. Cantello, Evidence of Pre-Synaptic Dopaminergic Deficit in a Patient with a Novel Progranulin $\mathrm{Mu}-$ tation Presenting with Atypical Parkinsonism (4) 747-752

Carnicelli, L., see Bonakis, A. (1) 85-91

Caruana, L., see Ghidoni, R. (3) 533-539

Carvalho, C., P.S. Katz, S. Dutta, P.V.G. Katakam, P.I. Moreira and D.W. Busija, Increased Susceptibility to Amyloid- $\beta$ Toxicity in Rat Brain Microvascular Endothelial Cells under Hyperglycemic Conditions (1) 75-83

Castelli, D., see Serra, L. (2) 391-402

Ceballos, C., see Ren, Y. (3) 589-600

Cercignani, M., see Serra, L. (2) 391-402

Cereda , C., see Benussi, L. (4) 799-808

Cestari, J.A.F., see de Souza Rolim, T. (4) 823-829

Chakrabarti, A., K. Roy and D. Mukhopadhyay, Differential Expression of Neuroblastoma Cellular Proteome due to AICD Overexpression (4) 845-855

Chakrabarti, O., see Roy, K. (4) 881-895

Chang, K.-T., see Lee, Y. (2) 251-267

Chen, E.S., see Silva, P.N. (1) 165-170

Cheng, Y., see Zhang, C. (2) 269-280

Chien, D.T., A.K. Szardenings, S. Bahri, J.C. Walsh, F. Mu, C. Xia, W.R. Shankle, A.J. Lerner, M.-Y. Su, A. Elizarov and H.C. Kolb, Early Clinical PET Imaging Results with the Novel PHF-Tau Radioligand [F18]-T808 (1) 171-184
Chong, Y.F.V., see Diaz-de-Grenu, L.Z. (3) 647-659

Cid-Fernández, S., M. Lindín and F. Díaz, Effects of Amnestic Mild Cognitive Impairment on N2 and P3 Go/NoGo ERP Components (2) 295-306

Ciotti, M.T., see Severini, C. (2) 281-293

Cirrito, J.R., see Paganetti, P. (4) 767-786

Clarke, D., see Parham, C. (2) 415-423

Clément, S., see Narme, P. (2) 359-369

Clerici, M., see Mancuso, R. (4) 741-745

Clerici, M., see Saresella, M. (2) 403-413

Clodomiro, A., see Anfossi, M. (2) 351-357

Colao, R., see Anfossi, M. (2) 351-357

Colelli, T., see Percy, M. (4) 907-922

Comi, C., see Carecchio, M. (4) 747-752

Conidi, M.E., see Anfossi, M. (2) 351-357

Constam, D., see Tzanoulinou, S. (4) 951-964

Coscio, E.D., see Bonakis, A. (1) 85-91

Costa, M., see Milojevic, J. (4) 753-765

Courtaigne, B., see Narme, P. (2) 359-369

Cummings, J., K. Zhong and C. Bernick, The Cleveland Clinic Lou Ruvo Center for Brain Health: Keeping Memory Alive (1) 103-109

Curcio, G., see Scrascia, F. (4) 939-950

Curcio, S.A.M., see Anfossi, M. (2) 351-357

Cusimano, M.D., see Di Ieva, A. (2) 331-350

D’ Onofrio, M., see Tzanoulinou, S. (4) 951-964

Daniilidou, M., see Lupton, M.K. (4) 897-906

Davis, J., see Xu, W. (3) 621-632

De Deyn, P.P., see Van der Mussele, S. (2) 319-329

de Siqueira, J.T.T., see de Souza Rolim, T. (4) 823-829

de Siqueira, S.R.D.T., see de Souza Rolim, T. (4) 823829

de Souza Rolim, T., G.M.C. Fabri, R. Nitrini, R. Anghinah, M.J. Teixeira, J.T.T. de Siqueira, J.A.F. Cestari and S.R.D.T. de Siqueira, Oral Infections and Orofacial Pain in Alzheimer's Disease: A CaseControl Study (4) 823-829

de Wilde, M.C., see van Wijk, N. (3) 459-479

Devraj, K., see Paganetti, P. (4) 767-786

Dhuyvetter, D., see Borghys, H. (1) 39-48

Di Ieva, A., M. Valli and M.D. Cusimano, Distinguishing Alzheimer's Disease from Normal Pressure Hydrocephalus: A Search for MRI Biomarkers (2) 331-350

Di Lorenzo, R., see Anfossi, M. (2) 351-357

Di Matteo, A., see Severini, C. (2) 281-293

Díaz, F., see Cid-Fernández, S. (2) 295-306

Diaz-de-Grenu, L.Z., J. Acosta-Cabronero, Y.F.V. Chong, J.M.S. Pereira, S.A. Sajjadi, G.B. Williams and P.J. Nestor, A Brief History of Voxel-Based 
Grey Matter Analysis in Alzheimer's Disease (3) 647-659

Dickson, D.W., see Ren, Y. (3) 589-600

Dillen, L., see Borghys, H. (1) 39-48

Dobson, R.J.B., see Kiddle, S.J. (3) 515-531

Dodge, H.H., see Shinto, L. (1) 111-120

Doecke, J., see Squitti, R. (4) 809-822

Drótos, G., see Pákáski, M. (2) 371-377

Duan, Y., see Wang, J. (2) 245-250

Dutta, S., see Carvalho, C. (1) 75-83

Economou, N.-T., see Bonakis, A. (1) 85-91

Ehrlé, N., see Narme, P. (2) 359-369

Elashoff, D., see Grill, J.D. (3) 507-514

Elizarov, A., see Chien, D.T. (1) 171-184

Enciu, A.-M., see Gheorghiu, M. (4) 787-798

Engelborghs, S., see Van der Mussele, S. (2) 319-329

Evgen'ev, M., see Bobkova, N.V. (2) 425-435

Fabri, G.M.C., see de Souza Rolim, T. (4) 823-829

Fadda, L., see Serra, L. (2) 391-402

Fallgatter, A.J., see Müller, S. (2) 379-390

Falsetti, L., see Buratti, L. (2) 445-453

Fazekas, Ö.C., see Pákáski, M. (2) 371-377

Fehér, Á., see Pákáski, M. (2) 371-377

Feng, X., see Wang, J. (2) 245-250

Fenoglio, C., see Carecchio, M. (4) 747-752

Flocco, R., see Benussi, L. (4) 799-808

Flocco, R., see Ghidoni, R. (3) 533-539

Florenzano, F., see Severini, C. (2) 281-293

Forloni, G., see Benussi, L. (4) 799-808

Fostinelli, S., see Benussi, L. (4) 799-808

Fox, N., see Taher, N. (4) 831-844

Frangipane, F., see Anfossi, M. (2) 351-357

Froestl, W., see Paganetti, P. (4) 767-786

Funaki, M., see Ito, S. (1) 185-200

Furuya, T.K., see Silva, P.N. (1) 165-170

Gai, W.-P., see Yan, X.-X. (4) 705-718

Gainotti, G., D. Quaranta, M.G. Vita and C. Marra, Neuropsychological Predictors of Conversion from Mild Cognitive Impairment to Alzheimer's Disease (3) 481-495

Galimberti, D., see Carecchio, M. (4) 747-752

Gallo, M., see Anfossi, M. (2) 351-357

Gallucci, M., A. Zanardo, M. Bendini, F.D. Paola, P. Boldrini and E. Grossi, Serum Folate, Homocysteine, Brain Atrophy, and Auto-CM System: The Treviso Dementia (TREDEM) Study (3) 581587

Galvan, V., see Sun, Y.-X. (2) 437-444
Garbuz, D.G., see Bobkova, N.V. (2) 425-435

Garcia, A., see Percy, M. (4) 907-922

Garrett, R., see Taher, N. (4) 831-844

Gatti, A., see Saresella, M. (2) 403-413

Gheorghiu, E., see Gheorghiu, M. (4) 787-798

Gheorghiu, M., A.-M. Enciu, B.O. Popescu and E. Gheorghiu, Functional and Molecular Characterization of the Effect of Amyloid- $\beta_{42}$ on an in vitro Epithelial Barrier Model (4) 787-798

Ghidoni, R., R. Flocco, A. Paterlini, M. Glionna, L. Caruana, E. Tonoli, G. Binetti and L. Benussi, Secretory Leukocyte Protease Inhibitor Protein Regulates the Penetrance of Frontotemporal Lobar Degeneration in Progranulin Mutation Carriers (3) 533-539

Ghidoni, R., see Benussi, L. (4) 799-808

Gijsen, H., see Borghys, H. (1) 39-48

Glionna, M., see Benussi, L. (4) 799-808

Glionna, M., see Ghidoni, R. (3) 533-539

Goeman, J., see Van der Mussele, S. (2) 319-329

Grant, W.B., Trends in Diet and Alzheimer's Disease During the Nutrition Transition in Japan and Developing Countries (3) 611-620

Greenberg, D.A., see Sun, Y.-X. (2) 437-444

Grill, J.D., Y. Zhou, J. Karlawish and D. Elashoff, Does Study Partner Type Impact the Rate of Alzheimer's Disease Progression? (3) 507-514

Groenendijk, M., see van Wijk, N. (3) 459-479

Grossi, E., see Gallucci, M. (3) 581-587

Guan, H.-S., see Tan, M.-S. (3) 633-646

Guglielmotti, A., see Severini, C. (2) 281-293

Hageman, R.J.J., see van Wijk, N. (3) 459-479

Hamilton, G., see Lupton, M.K. (4) 897-906

Han, P., see Shi, J. (1) 1-9

Hautzinger, M., see Müller, S. (2) 379-390

Havas, D., see Paganetti, P. (4) 767-786

Heringa, S.M., Y.D. Reijmer, A. Leemans, H.L. Koek, L.J. Kappelle and G.J. Biessels on behalf of the Utrecht Vascular Cognitive Impairment (VCI) Study Group, Multiple Microbleeds are Related to Cerebral Network Disruptions in Patients with Early Alzheimer's Disease (1) 211-221

Hernis, A., see Mancuso, R. (4) 741-745

Herukka, S.-K., see Lundström, S.L. (3) 567-579

Hickie, I.B., see Naismith, S.L. (4) 867-879

Hicks, M., see Percy, M. (4) 907-922

Ho, V., see Percy, M. (4) 907-922

Hodges, A., see Kiddle, S.J. (3) 515-531

Hodges, J.R., see Kamminga, J. (3) 669-679

Hodges, J.R., see Naismith, S.L. (4) 867-879 
Hoffmeyer, A., see Kirste, T. (1) 121-132

Hohman, T.J., see Koran, M.E.I. (1) 145-154

Hong, Y., see Lee, Y. (2) 251-267

Honjo, Y., T. Horibe, A. Torisawa, H. Ito, A. Nakanishi, H. Mori, T. Komiya, R. Takahashi and K. Kawakami, Protein Disulfide Isomerase P5-Immunopositive Inclusions in Patients with Alzheimer's Disease (3) 601-609

Horibe, T., see Honjo, Y. (3) 601-609

Hu, N., M.-S. Tan, J.-T. Yu, L. Sun, L. Tan, Y.-L. Wang, T. Jiang and L. Tan, Increased Expression of TREM2 in Peripheral Blood of Alzheimer's Disease Patients (3) 497-501

Huh, J.-W., see Lee, Y. (2) 251-267

Hyman, B.T., see Ren, Y. (3) 589-600

Irish, M., see Kamminga, J. (3) 669-679

Isaacs, G.D., see Taher, N. (4) 831-844

Ito, H., see Honjo, Y. (3) 601-609

Ito, S., S. Ohtsuki, S. Murata, Y. Katsukura, H. Suzuki, M. Funaki, M. Tachikawa and T. Terasaki, Involvement of Insulin-Degrading Enzyme in Insulin- and Atrial Natriuretic Peptide-Sensitive Internalization of Amyloid- $\beta$ Peptide in Mouse Brain Capillary Endothelial Cells (1) 185-200

Jacobs, T., see Borghys, H. (1) 39-48

Janka, Z., see Pákáski, M. (2) 371-377

Jeong, K.-J., see Lee, Y. (2) 251-267

Ji, X., see Sun, Y.-X. (2) 437-444

Jia, J., see Sun, Y.-X. (2) 437-444

Jiang, A., see Percy, M. (4) 907-922

Jiang, T., see Hu, N. (3) 497-501

Jiang, T., see Tan, M.-S. (3) 633-646

Jin, K., see Sun, Y.-X. (2) 437-444

Jin, L.-W., see Wang, J. (2) 245-250

Johnston, C., see Kiddle, S.J. (3) 515-531

Jordán, J., see Cano-Cuenca, N. (1) 155-164

Jorquera, J.I., see Milojevic, J. (4) 753-765

Joshi, Y.B., A.D. Meco and D. Praticó, Modulation of Amyloid- $\beta$ Production by Leukotriene $\mathrm{B}_{4}$ via the $\gamma$-Secretase Pathway (3) 503-506

Juha Laurén, Cellular Prion Protein as a Therapeutic Target in Alzheimer's Disease (2) 227-244

Juhász, A., see Pákáski, M. (2) 371-377

Kaerst, L., A. Kuhlmann, D. Wedekind, K. Stoeck, P. Lange and I. Zerr, Using Cerebrospinal Fluid Marker Profiles in Clinical Diagnosis of Dementia with Lewy Bodies, Parkinson's Disease, and Alzheimer's Disease (1) 63-73
Kálmán, J., see Pákáski, M. (2) 371-377

Kamminga, J., C. O’Callaghan, J.R. Hodges and M. Irish, Differential Prospective Memory Profiles in Frontotemporal Dementia Syndromes (3) 669-679

Kamphuis, P.J.G.H., see van Wijk, N. (3) 459-479

Kappelle, L.J., see Heringa, S.M. (1) 211-221

Kåreholt, I., see Tolppanen, A.-M. (1) 201-209

Karlawish, J., see Grill, J.D. (3) 507-514

Karpov, V., see Bobkova, N.V. (2) 425-435

Katakam, P.V.G., see Carvalho, C. (1) 75-83

Katsukura, Y., see Ito, S. (1) 185-200

Katz, P.S., see Carvalho, C. (1) 75-83

Kawakami, K., see Honjo, Y. (3) 601-609

Kaye, J., see Shinto, L. (1) 111-120

Keitel, S., see Wirz, K.T.S. (4) 719-740

Kermer, P., see Schmitz, M. (3) 551-565

Kiddle, S.J., M. Sattlecker, P. Proitsi, A. Simmons, E. Westman, C. Bazenet, S.K. Nelson, S. Williams, A. Hodges, C. Johnston, H. Soininen, I. Kłoszewska, P. Mecocci, M. Tsolaki, B. Vellas, S. Newhouse, S. Lovestone and R.J.B. Dobson, Candidate Blood Proteome Markers of Alzheimer's Disease Onset and Progression: A Systematic Review and Replication Study (3) 515-531

Kieran, D., see Paganetti, P. (4) 767-786

Kim, J.-S., see Lee, Y. (2) 251-267

Kim, S.-H., see Lee, Y. (2) 251-267

Kim, S.-U., see Lee, Y. (2) 251-267

Kim, Y.-H., see Lee, Y. (2) 251-267

Kinosian, B., see Razlighi, Q.R. (3) 661-668

Kirste, T., A. Hoffmeyer, P. Koldrack, A. Bauer, S. Schubert, S. Schröder and S. Teipel, Detecting the Effect of Alzheimer's Disease on Everyday Motion Behavior (1) 121-132

Kitaygorodsky, J., see Percy, M. (4) 907-922

Kivipelto, M., see Tolppanen, A.-M. (1) 201-209

Kłoszewska, I., see Kiddle, S.J. (3) 515-531

Koek, H.L., see Heringa, S.M. (1) 211-221

Kolasa, K., see Nelson, A.R. (4) 867-879

Kolb, H.C., see Chien, D.T. (1) 171-184

Koldrack, P., see Kirste, T. (1) 121-132

Kolosova, N.G., see Stefanova, N.A. (3) 681-694

Komiya, T., see Honjo, Y. (3) 601-609

Koran, M.E.I., T.J. Hohman, S.A. Meda and T.A. Thornton-Wells for the Alzheimer's Disease Neuroimaging Initiative, Genetic Interactions within Inositol-Related Pathways are Associated with Longitudinal Changes in Ventricle Size (1) 145-154

Kosuri, P., see Lee, L. (1) 49-62

Kotarba, A.E., see Xu, W. (3) 621-632 
Kovács, J., see Pákáski, M. (2) 371-377

Ktonas, P., see Bonakis, A. (1) 85-91

Kuhlmann, A., see Kaerst, L. (1) 63-73

Kukharsky, M.S., see Bobkova, N.V. (2) 425-435

Kuniyoshi, S.M., see Shi, J. (1) 1-9

Laatikainen, T., see Tolppanen, A.-M. (1) 201-209

Labio, R.W., see Silva, P.N. (1) 165-170

Lange, P., see Kaerst, L. (1) 63-73

Lee, K.-M., see Lee, Y. (2) 251-267

Lee, L., P. Kosuri and O. Arancio, Picomolar Amyloid$\beta$ Peptides Enhance Spontaneous Astrocyte Calcium Transients (1) 49-62

Lee, S.-R., see Lee, Y. (2) 251-267

Lee, Y., Y.-H. Kim, S.-J. Park, J.-W. Huh, S.-H. Kim, S.-U. Kim, J.-S. Kim, K.-J. Jeong, K.-M. Lee, Y. Hong, S.-R. Lee and K.-T. Chang, Insulin/IGF Signaling-Related Gene Expression in the Brain of a Sporadic Alzheimer's Disease Monkey Model Induced by Intracerebroventricular Injection of Streptozotocin (2) 251-267

Leemans, A., see Heringa, S.M. (1) 211-221

Lei, H., see Wang, J. (2) 245-250

Lerner, A.J., see Chien, D.T. (1) 171-184

Lewis, S.J.G., see Naismith, S.L. (4) 867-879

Leyhe, T. see Müller, S. (2) 379-390

Li, S.-Q., see Yu, Y. (1)133-144

Liang, H.-F., see Sun, S.-W. (1) 93-101

Liebner, S., see Paganetti, P. (4) 767-786

Lin, K., see Lupton, M.K. (4) 897-906

Lin, W.-L., see Ren, Y. (3) 589-600

Lindín, M., see Cid-Fernández, S. (2) 295-306

Liu, J., see Yu, Y. (1)133-144

Lovestone, S., see Kiddle, S.J. (3) 515-531

Lundström, S.L., H. Yang, Y. Lyutvinskiy, D. Rutishauser, S.-K. Herukka, H. Soininen and R.A. Zubarev, Blood Plasma IgG Fc Glycans are Significantly Altered in Alzheimer's Disease and Progressive Mild Cognitive Impairment (3) 567579

Luo, X.-G., see Yan, X.-X. (4) 705-718

Lupton, M.K., P. Proitsi, K. Lin, G. Hamilton, M. Daniilidou, M. Tsolaki and J.F. Powell, The Role of ABCA1 Gene Sequence Variants on Risk of Alzheimer's Disease (4) 897-906

Luzzi, S., see Buratti, L. (2) 445-453

Lyutvinskiy, Y., see Lundström, S.L. (3) 567-579

Ma, C., see Yan, X.-X. (4) 705-718

Madani, R., see Paganetti, P. (4) 767-786

Maestri, M., see Bonakis, A. (1) 85-91
Makovac, E., see Serra, L. (2) 391-402

Maletta, R., see Anfossi, M. (2) 351-357

Mancuso, R., F. Baglio, M. Cabinio, E. Calabrese, A. Hernis, R. Nemni and M. Clerici, Titers of Herpes Simplex Virus Type 1 Antibodies Positively Correlate with Grey Matter Volumes in Alzheimer's Disease (4) 741-745

Mao, X., see Sun, Y.-X. (2) 437-444

Mariën, P., see Van der Mussele, S. (2) 319-329

Marra, C., see Gainotti, G. (3) 481-495

Marra, C., see Serra, L. (2) 391-402

Maruszak, A., A. Pilarski, T. Murphy, N. Branch and S. Thuret, Hippocampal Neurogenesis in Alzheimer's Disease: Is There a Role for Dietary Modulation? (1) 11-38

Marventano, I., see Saresella, M. (2) 403-413

McKenzie, C., see Taher, N. (4) 831-844

McMahon, L.L., see Nelson, A.R. (4) 867-879

Mechawar, N., see Silva, P.N. (1) 165-170

Meco, A.D., see Joshi, Y.B. (3) 503-506

Mecocci, P., see Kiddle, S.J. (3) 515-531

Meda, S.A., see Koran, M.E.I. (1) 145-154

Medvinskaya, N., see Bobkova, N.V. (2) 425-435

Mei, J., see Sun, S.-W. (1) 93-101

Melacini, G., see Milojevic, J. (4) 753-765

Melgari, J.-M., see Scrascia, F. (4) 939-950

Mercanti, D., see Severini, C. (2) 281-293

Mercken, M., see Borghys, H. (1) 39-48

Migliore, S., see Scrascia, F. (4) 939-950

Mill, J., see Silva, P.N. (1) 165-170

Milojevic, J., M. Costa, A.M. Ortiz, J.I. Jorquera and G. Melacini, In Vitro Amyloid- $\beta$ Binding and Inhibition of Amyloid- $\beta$ Self-Association by Therapeutic Albumin (4) 753-765

Mirabelli, M., see Anfossi, M. (2) 351-357

Montine, T., see Shinto, L. (1) 111-120

Moreira, P.I., see Carvalho, C. (1) 75-83

Mori, H., see Honjo, Y. (3) 601-609

$\mathrm{Mu}, \mathrm{F}$., see Chien, D.T. (1) 171-184

Muhs, A., see Paganetti, P. (4) 767-786

Mukhopadhyay, D., see Chakrabarti, A. (4) 845-855

Mukhopadhyay, D., see Roy, K. (4) 881-895

Müller, S., C. Mychajliw, M. Hautzinger, A.J. Fallgatter, R. Saur and T. Leyhe, Memory for Past Public Events Depends on Retrieval Frequency but not Memory Age in Alzheimer's Disease (2) 379-390

Munsch, F., see Narme, P. (2) 359-369

Muraleva, N.A., see Stefanova, N.A. (3) 681-694

Murata, S., see Ito, S. (1) 185-200

Murphy, T., see Maruszak, A. (1) 11-38

Mychajliw, C., see Müller, S. (2) 379-390 
Naismith, S.L., I.B. Hickie, Z. Terpening, S.W. Rajaratnam, J.R. Hodges, S. Bolitho, N.L. Rogers and S.J.G. Lewis, Circadian Misalignment and Sleep Disruption in Mild Cognitive Impairment (4) 867879

Nakanishi, A., see Honjo, Y. (3) 601-609

Narme, P., S. Clément, N. Ehrlé, L. Schiaratura, S. Vachez, B. Courtaigne, F. Munsch and S. Samson, Efficacy of Musical Interventions in Dementia: Evidence from a Randomized Controlled Trial (2) 359-369

Nelson, A.R., K. Kolasa and L.L. McMahon, Noradrenergic Sympathetic Sprouting and Cholinergic Reinnervation Maintains Non-Amyloidogenic Processing of AßPP (4) 867-879

Nelson, S.K., see Kiddle, S.J. (3) 515-531

Nemni, R., see Mancuso, R. (4) 741-745

Nemni, R., see Saresella, M. (2) 403-413

Nesterova, I., see Bobkova, N.V. (2) 425-435

Nestor, P.J., see Diaz-de-Grenu, L.Z. (3) 647-659

Newhouse, S., see Kiddle, S.J. (3) 515-531

Ngandu, T., see Tolppanen, A.-M. (1) 201-209

Ninkina, N.N., see Bobkova, N.V. (2) 425-435

Nitrini, R., see de Souza Rolim, T. (4) 823-829

Nostrand, W.E.V., see Xu, W. (3) 621-632

Nudler, E., see Bobkova, N.V. (2) 425-435

O’Callaghan, C., see Kamminga, J. (3) 669-679

Ohtsuki, S., see Ito, S. (1) 185-200

Ortiz, A.M., see Milojevic, J. (4) 753-765

Pachter, J., see Severini, C. (2) 281-293

Paganetti, P., K. Antoniello, K. Devraj, N. Toni, D. Kieran, R. Madani, M. Pihlgren, O. Adolfsson, W. Froestl, A. Schrattenholz, S. Liebner, D. Havas, M. Windisch, J.R. Cirrito, A. Pfeifer and A. Muhs, Increased Efflux of Amyloid- $\beta$ Peptides through the Blood-Brain Barrier by Muscarinic Acetylcholine Receptor Inhibition Reduces Pathological Phenotypes in Mouse Models of Brain Amyloidosis (4) 767-786

Pákáski, M., Á. Fehér, A. Juhász, G. Drótos, Ö.C. Fazekas, J. Kovács, Z. Janka and J. Kálmán, Serum Adipokine Levels Modified by Donepezil Treatment in Alzheimer's Disease (2) 371-377

Pantieri, R., see Benussi, L. (4) 799-808

Paola, F.D., see Gallucci, M. (3) 581-587

Papageorgiou, S.G., see Bonakis, A. (1) 85-91

Paparrigopoulos, T., see Bonakis, A. (1) 85-91

Parham, C., L. Auckland, J. Rachwal, D. Clarke and G. Bix, Perlecan Domain V Inhibits Amyloid- $\beta$
Induced Brain Endothelial Cell Toxicity and Restores Angiogenic Function (2) 415-423

Park, S.-J., see Lee, Y. (2) 251-267

Parpia, A., see Percy, M. (4) 907-922

Pasqualetti, P., see Squitti, R. (4) 809-822

Passeri, P.P., see Severini, C. (2) 281-293

Paterlini, A., see Benussi, L. (4) 799-808

Paterlini, A., see Ghidoni, R. (3) 533-539

Payão, S.L., see Silva, P.N. (1) 165-170

Peng, L., see Yu, Y. (1)133-144

Percy, M., M.J. Somerville, M. Hicks, T. Colelli, E. Wright, J. Kitaygorodsky, A. Jiang, V. Ho, A. Parpia, M.K. Wong and A. Garcia, Risk Factors for Development of Dementia in a Unique SixYear Cohort Study. I. An Exploratory, Pilot Study of Involvement of the E4 Allele of Apolipoprotein E, Mutations of the Hemochromatosis-HFE Gene, Type 2 Diabetes, and Stroke (4) 907-922

Pereira, J.M.S., see Diaz-de-Grenu, L.Z. (3) 647-659

Perri, R., see Serra, L. (2) 391-402

Peters, F., S. Villeneuve and S. Belleville, Predicting Progression to Dementia in Elderly Subjects with Mild Cognitive Impairment Using Both Cognitive and Neuroimaging Predictors (2) 307-318

Pfeifer, A., see Paganetti, P. (4) 767-786

Piancone, F., see Saresella, M. (2) 403-413

Piccoli, E., see Benussi, L. (4) 799-808

Pihlgren, M., see Paganetti, P. (4) 767-786

Pilarski, A., see Maruszak, A. (1) 11-38

Pitocco, F., see Scrascia, F. (4) 939-950

Polydoro, M., see Ren, Y. (3) 589-600

Popescu, B.O., see Gheorghiu, M. (4) 787-798

Possenti, R., see Severini, C. (2) 281-293

Powell, J.F., see Lupton, M.K. (4) 897-906

Pradier, C., see Tifratene, K. (3) 541-549

Praticó, D., see Joshi, Y.B. (3) 503-506

Proitsi, P., see Kiddle, S.J. (3) 515-531

Proitsi, P., see Lupton, M.K. (4) 897-906

Provinciali, L., see Buratti, L. (2) 445-453

Puccio, G., see Anfossi, M. (2) 351-357

Quaranta, D., see Gainotti, G. (3) 481-495

Quattrocchi, C.C., see Scrascia, F. (4) 939-950

Quinn, J., see Shinto, L. (1) 111-120

Quintiliani, L., see Scrascia, F. (4) 939-950

Rachwal, J., see Parham, C. (2) 415-423

Rainone, V., see Saresella, M. (2) 403-413

Rajaratnam, S.W., see Naismith, S.L. (4) 867-879

Rasmussen, L.T., see Silva, P.N. (1) 165-170

Raychaudhuri, M., see Roy, K. (4) 881-895 
Razlighi, Q.R., E. Stallard, J. Brandt, D. Blacker, M. Albert, N. Scarmeas, B. Kinosian, A.I. Yashin and Y. Stern, A New Algorithm for Predicting Time to Disease Endpoints in Alzheimer's Disease Patients (3) 661-668

Reijmer, Y.D., see Heringa, S.M. (1) 211-221

Rembach, A., see Squitti, R. (4) 809-822

Ren, Y., W.-L. Lin, L. Sanchez, C. Ceballos, M. Polydoro, T.L. Spires-Jones, B.T. Hyman, D.W. Dickson and N. Sahara, Endogenous Tau Aggregates in Oligodendrocytes of rTg4510 Mice Induced by Human P301L Tau (3) 589-600

Robert, P., see Tifratene, K. (3) 541-549

Robinson, J.K., see Xu, W. (3) 621-632

Rogers, N.L., see Naismith, S.L. (4) 867-879

Rossi, G., see Benussi, L. (4) 799-808

Rouis, A., see Tifratene, K. (3) 541-549

Roy, K., M. Raychaudhuri, O. Chakrabarti and D. Mukhopadhyay, Growth Factor Receptor-Bound Protein 2 Promotes Autophagic Removal of Amyloid- $\beta$ Protein Precursor Intracellular Domain Overload in Neuronal Cells (4) 881-895

Roy, K., see Chakrabarti, A. (4) 845-855

Rusanen, M., see Tolppanen, A.-M. (1) 201-209

Rutishauser, D., see Lundström, S.L. (3) 567-579

Saerens, J., see Van der Mussele, S. (2) 319-329

Sahara, N., see Ren, Y. (3) 589-600

Sajjadi, S.A., see Diaz-de-Grenu, L.Z. (3) 647-659

Sakarovitch, C., see Tifratene, K. (3) 541-549

Samokhin, A., see Bobkova, N.V. (2) 425-435

Samson, S., see Narme, P. (2) 359-369

Sanchez, L., see Ren, Y. (3) 589-600

Sandi, C., see Tzanoulinou, S. (4) 951-964

Saresella, M., I. Marventano, E. Calabrese, F. Piancone, V. Rainone, A. Gatti, M. Alberoni, R. Nemni and M. Clerici, A Complex Proinflammatory Role for Peripheral Monocytes in Alzheimer's Disease (2) 403-413

Sattlecker, M., see Kiddle, S.J. (3) 515-531

Saur, R., see Müller, S. (2) 379-390

Scarmeas, N., see Razlighi, Q.R. (3) 661-668

Scarpini, E., see Carecchio, M. (4) 747-752

Schiaratura, L., see Narme, P. (2) 359-369

Schmitz, M., K. Wulf, S.C. Signore, W.J. SchulzSchaeffer, P. Kermer, M. Bähr, F.S. Wouters, S. Zafar and I. Zerr, Impact of the Cellular Prion Protein on Amyloid- $\beta$ and 3PO-Tau Processing (3) 551-565

Schrattenholz, A., see Paganetti, P. (4) 767-786

Schröder, S., see Kirste, T. (1) 121-132
Schubert, S., see Kirste, T. (1) 121-132

Schulz-Schaeffer, W.J., see Schmitz, M. (3) 551-565

Scrascia, F., G. Curcio, F. Ursini, L. Trotta, L. Quintiliani, S. Migliore, C. Altamura, F. Pitocco, R. Altavilla, J.-M. Melgari, C.C. Quattrocchi and F. Vernieri, Relationship among Diffusion Tensor Imaging, EEG Activity, and Cognitive Status in Mild Cognitive Impairment and Alzheimer's Disease Patients (4) 939-950

Serpente, M., see Carecchio, M. (4) 747-752

Serra, L., L. Fadda, R. Perri, B. Spanò, C. Marra, D. Castelli, M. Torso, E. Makovac, M. Cercignani, C. Caltagirone and M. Bozzali, Constructional Apraxia as a Distinctive Cognitive and Structural Brain Feature of Pre-Senile Alzheimer's Disease (2) 391-402

Severini, C., P.P. Passeri, M.T. Ciotti, F. Florenzano, R. Possenti, C. Zona, A. Di Matteo, A. Guglielmotti, P. Calissano, J. Pachter and D. Mercanti, Bindarit, Inhibitor of CCL2 Synthesis, Protects Neurons Against Amyloid- $\beta$-Induced Toxicity (2) 281-293

Shakuf, V., see Ben-David, B.M. (4) 923-938

Shankle, W.R., see Chien, D.T. (1) 171-184

Shi, J., P. Han and S.M. Kuniyoshi, Cognitive Impairment in Neurological Diseases: Lessons from Apolipoprotein E (1) 1-9

Shi, W.-X., see Sun, S.-W. (1) 93-101

Shinto, L., J. Quinn, T. Montine, H.H. Dodge, W. Woodward, S. Baldauf-Wagner, D. Waichunas, L. Bumgarner, D. Bourdette, L. Silbert and J. Kaye, A Randomized Placebo-Controlled Pilot Trial of Omega-3 Fatty Acids and Alpha Lipoic Acid in Alzheimer's Disease (1) 111-120

Signore, S.C., see Schmitz, M. (3) 551-565

Sijben, J.W.C., see van Wijk, N. (3) 459-479

Silbert, L., see Shinto, L. (1) 111-120

Silva, P.N., T.K. Furuya, I.L. Braga, L.T. Rasmussen, R.W. Labio, P.H. Bertolucci, E.S. Chen, G. Turecki, N. Mechawar, S.L. Payão, J. Mill and M.C. Smith, Analysis of HSPA8 and HSPA9 mRNA Expression and Promoter Methylation in the Brain and Blood of Alzheimer's Disease Patients (1) 165-170

Silvestrini, M., see Buratti, L. (2) 445-453

Simmons, A., see Kiddle, S.J. (3) 515-531

Simonelli, I., see Squitti, R. (4) 809-822

Siotto, M., see Squitti, R. (4) 809-822

Skulachev, V.P., see Stefanova, N.A. (3) 681-694

Smirne, N., see Anfossi, M. (2) 351-357

Smirnov, A.A., see Bobkova, N.V. (2) 425-435

Smith, M.C., see Silva, P.N. (1) 165-170 
Soininen, H., see Kiddle, S.J. (3) 515-531

Soininen, H., see Lundström, S.L. (3) 567-579

Soininen, H., see Tolppanen, A.-M. (1) 201-209

Solís-García del Pozo, J.E., see Cano-Cuenca, N. (1) 155-164

Somers, N., see Van der Mussele, S. (2) 319-329

Somerville, M.J., see Percy, M. (4) 907-922

Spanò, B., see Serra, L. (2) 391-402

Spires-Jones, T.L., see Ren, Y. (3) 589-600

Squitti, R., I. Simonelli, M. Ventriglia, M. Siotto, P. Pasqualetti, A. Rembach, J. Doecke and A.I. Bush, Meta-Analysis of Serum Non-Ceruloplasmin Copper in Alzheimer's Disease (4) 809-822

Stallard, E., see Razlighi, Q.R. (3) 661-668

Stefanova, N.A., N.A. Muraleva, V.P. Skulachev and N.G. Kolosova, Alzheimer's Disease-Like Pathology in Senescence-Accelerated OXYS Rats can be Partially Retarded with Mitochondria-Targeted Antioxidant SkQ1 (3) 681-694

Stern, Y., see Razlighi, Q.R. (3) 661-668

Stoeck, K., see Kaerst, L. (1) 63-73

$\mathrm{Su}$, M.-Y., see Chien, D.T. (1) 171-184

Sun, L., see Hu, N. (3) 497-501

Sun, S.-W., H.-F. Liang, J. Mei, D. Xu and W.-X. Shi, In vivo Diffusion Tensor Imaging of Amyloid- $\beta$-Induced White Matter Damage in Mice (1) 93-101

Sun, Y.-X., X. Ji, X. Mao, L. Xie, J. Jia, V. Galvan, D.A. Greenberg and K. Jin, Differential Activation of mTOR Complex 1 Signaling in Human Brain with Mild to Severe Alzheimer's Disease (2) 437-444

Suzuki, H., see Ito, S. (1) 185-200

Swaab, D.F., see Wirz, K.T.S. (4) 719-740

Szardenings, A.K., see Chien, D.T. (1) 171-184

Tachikawa, M., see Ito, S. (1) 185-200

Tagliavini, F., see Benussi, L. (4) 799-808

Taher, N., C. McKenzie, R. Garrett, M. Baker, N. Fox and G.D. Isaacs, Amyloid- $\beta$ Alters the DNA Methylation Status of Cell-fate Genes in an Alzheimer's Disease Model (4) 831-844

Takahashi, R., see Honjo, Y. (3) 601-609

Tan, L., see Hu, N. (3) 497-501

Tan, L., see Hu, N. (3) 497-501

Tan, L., see Tan, M.-S. (3) 633-646

Tan, M.-S., J.-T. Yu, T. Jiang, X.-C. Zhu, H.-S. Guan and L. Tan, IL12/23 p40 Inhibition Ameliorates Alzheimer's Disease-Associated Neuropathology and Spatial Memory in SAMP8 Mice (3) 633-646

Tan, M.-S., see Hu, N. (3) 497-501

Teipel, S., see Kirste, T. (1) 121-132

Teixeira, M.J., see de Souza Rolim, T. (4) 823-829
Terasaki, T., see Ito, S. (1) 185-200

Terazzi, E., see Carecchio, M. (4) 747-752

Terpening, Z., see Naismith, S.L. (4) 867-879

Tewari, A., see Ben-David, B.M. (4) 923-938

Theodoropoulos, P., see Bonakis, A. (1) 85-91

Thuret, S., see Maruszak, A. (1) 11-38

Tifratene, K., C. Sakarovitch, A. Rouis, C. Pradier and P. Robert the participating centers, Mild Cognitive Impairment and Anti-Alzheimer Disease Medications: A Cross Sectional Study of the French National Alzheimer Databank (BNA) (3) 541-549

Tolppanen, A.-M., T. Ngandu, I. Kåreholt, T. Laatikainen, M. Rusanen, H. Soininen and M. Kivipelto, Midlife and Late-Life Body Mass Index and LateLife Dementia: Results from a Prospective Population-Based Cohort (1) 201-209

Toni, N., see Paganetti, P. (4) 767-786

Tonoli, E., see Benussi, L. (4) 799-808

Tonoli, E., see Ghidoni, R. (3) 533-539

Torchia, G., see Anfossi, M. (2) 351-357

Torisawa, A., see Honjo, Y. (3) 601-609

Torso, M., see Serra, L. (2) 391-402

Trotta, L., see Scrascia, F. (4) 939-950

Tsolaki, M., see Kiddle, S.J. (3) 515-531

Tsolaki, M., see Lupton, M.K. (4) 897-906

Turecki, G., see Silva, P.N. (1) 165-170

Tzanoulinou, S., R. Brandi, I. Arisi, M. D’ Onofrio, S.M. Urfer, C. Sandi, D. Constam and S. Capsoni, Pathogen-Free Husbandry Conditions Alleviate Behavioral Deficits and Neurodegeneration in AD10 Anti-NGF Mice (4) 951-964

Urfer, S.M., see Tzanoulinou, S. (4) 951-964

Ursini, F., see Scrascia, F. (4) 939-950

Vachez, S., see Narme, P. (2) 359-369

Vagiakis, E., see Bonakis, A. (1) 85-91

Valli, M., see Di Ieva, A. (2) 331-350

Van der Mussele, S., P. Mariën, J. Saerens, N. Somers, J. Goeman, P.P. De Deyn and S. Engelborghs, Behavioral Syndromes in Mild Cognitive Impairment and Alzheimer's Disease (2) 319-329

Van Lieshout, P.H.H.M., see Ben-David, B.M. (4) 923938

van Wijk, N., L.M. Broersen, M.C. de Wilde, R.J.J. Hageman, M. Groenendijk, J.W.C. Sijben and P.J.G.H. Kamphuis, Targeting Synaptic Dysfunction in Alzheimer's Disease by Administering a Specific Nutrient Combination (3) 459-479

Vasso, F., see Anfossi, M. (2) 351-357

Vellas, B., see Kiddle, S.J. (3) 515-531 
Ventriglia, M., see Squitti, R. (4) 809-822

Verhaagen, J., see Wirz, K.T.S. (4) 719-740

Vernieri, F., see Scrascia, F. (4) 939-950

Villeneuve, S., see Peters, F. (2) 307-318

Vita, M.G., see Gainotti, G. (3) 481-495

Viticchi, G., see Buratti, L. (2) 445-453

Waichunas, D., see Shinto, L. (1) 111-120

Walsh, J.C., see Chien, D.T. (1) 171-184

Wang, C., see Zhang, C. (2) 269-280

Wang, H., see Zhang, C. (2) 269-280

Wang, J., X. Feng, Z. Bai, L.-W. Jin, Y. Duan and H. Lei, Chromosome 19p in Alzheimer's Disease: When Genome Meets Transcriptome (2) 245-250

Wang, Y.-L., see Hu, N. (3) 497-501

Wedekind, D., see Kaerst, L. (1) 63-73

Westman, E., see Kiddle, S.J. (3) 515-531

Williams, G.B., see Diaz-de-Grenu, L.Z. (3) 647-659

Williams, S., see Kiddle, S.J. (3) 515-531

Wilson, S.P., see Zhang, C. (2) 269-280

Windisch, M., see Paganetti, P. (4) 767-786

Wirz, K.T.S., S. Keitel, D.F. Swaab, J. Verhaagen and K. Bossers, Early Molecular Changes in Alzheimer Disease: Can We Catch the Disease in its Presymptomatic Phase? (4) 719-740

Wong, M.K., see Percy, M. (4) 907-922

Woodward, W., see Shinto, L. (1) 111-120

Wouters, F.S., see Schmitz, M. (3) 551-565

Wright, E., see Percy, M. (4) 907-922

Wulf, K., see Schmitz, M. (3) 551-565

Xia, C., see Chien, D.T. (1) 171-184

Xie, L., see Sun, Y.-X. (2) 437-444

Xu, D., see Sun, S.-W. (1) 93-101

Xu, F., see Xu, W. (3) 621-632
Xu, J., see Zhang, C. (2) 269-280

Xu, W., F. Xu, M.E. Anderson, A.E. Kotarba, J. Davis, J.K. Robinson and W.E.V. Nostrand, Cerebral Microvascular Rather than Parenchymal Amyloid- $\beta$ Protein Pathology Promotes Early Cognitive Impairment in Transgenic Mice (3) 621-632

Yan, X.-X., C. Ma, W.-P. Gai, H. Cai and X.-G. Luo, Can BACE1 Inhibition Mitigate Early Axonal Pathology in Neurological Diseases? (4) 705-718

Yang, H., see Lundström, S.L. (3) 567-579

Yashin, A.I. , see Razlighi, Q.R. (3) 661-668

Yashin, V., see Bobkova, N.V. (2) 425-435

Ye, R.D., see Yu, Y. (1)133-144

Yu, J.-T., see Hu, N. (3) 497-501

Yu, J.-T., see Tan, M.-S. (3) 633-646

Yu, Y., J. Liu, S.-Q. Li, L. Peng and R.D. Ye, Serum Amyloid A Differentially Activates Microglia and Astrocytes via the PI3K Pathway (1)133-144

Zafar, S., see Schmitz, M. (3) 551-565

Zanardo, A., see Gallucci, M. (3) 581-587

Zerr, I., see Kaerst, L. (1) 63-73

Zerr, I., see Schmitz, M. (3) 551-565

Zhang, C., Y. Cheng, H. Wang, C. Wang, S.P. Wilson, J. $\mathrm{Xu}$ and H.-T. Zhang, RNA Interference-Mediated Knockdown of Long-Form Phosphodiesterase-4D (PDE4D) Enzyme Reverses Amyloid- $\beta_{42}$-Induced Memory Deficits in Mice (2) 269-280

Zhang, H.-T., see Zhang, C. (2) 269-280

Zhong, K., see Cummings, J. (1) 103-109

Zhou, Y., see Grill, J.D. (3) 507-514

Zhu, X.-C., see Tan, M.-S. (3) 633-646

Zona, C., see Severini, C. (2) 281-293

Zubarev, R.A., see Lundström, S.L. (3) 567-579 